Article

\title{
Cytospyrone and Cytospomarin: Two New Polyketides Isolated from Mangrove Endophytic Fungus, Cytospora sp. ${ }^{\dagger}$
}

\author{
Chengwen Wei ${ }^{1}$, Qin Deng ${ }^{2}$, Mengyu Sun ${ }^{1}$ and Jing $X u^{1,2, *}$ \\ 1 Key Laboratory of Advanced Materials of Tropical Island Resources of Ministry of Education, \\ School of Chemical Engineering and Technology, Hainan University, Haikou 570228, China; \\ chengwen121412@163.com (C.W.); sunmengyu0305@163.com (M.S.) \\ 2 College of Horticulture and Landscape, Hainan University, Haikou 570228, China; dengqin_e@yeah.net \\ * Correspondence: happyjing3@hainanu.edu.cn; Tel.: +86-898-6627-9226; Fax: +86-898-6627-9010 \\ $\dagger$ Dedicated to Prof. Dr. Peter Proksch on His Research Career in Marine Natural Product Chemistry.
}

Academic Editor: Akihito Yokosuka

Received: 24 August 2020; Accepted: 10 September 2020; Published: 15 September 2020

\begin{abstract}
Two new polyketides, cytospyrone (1), cytospomarin (2), together with three known metabolites dimethoxyphtalide (3), integracin A (4) and integracin B (5), were isolated from the culture of Cytospora sp. from the Chinese mangrove Ceriops tagal. Their structures were elucidated by extensive spectroscopic analyses and time dependent density functional theory (TDDFT), calculation of electronic circular dichroism (ECD) and optical rotation (OR) data. Compound 2 displayed weak inhibitory activity against Escherichia coli GIM1.201 (minimum inhibitory concentration (MIC) value of $0.35 \mathrm{mM}$ ). Compounds 4 and 5 displayed significant cytotoxicity against human cancer cell line HepG2 ( $\mathrm{IC}_{50}$ values of $5.98 \pm 0.12 \mu \mathrm{M}$ and $9.97 \pm 0.06 \mu \mathrm{M}$, respectively), more potent than the positive control 5-fluorouracil ( $\mathrm{IC}_{50}$ value of $43.50 \pm 3.69 \mu \mathrm{M}$ ).
\end{abstract}

Keywords: Cytospora sp.; polyketides; structure identification; antimicrobial activity; cytotoxicity

\section{Introduction}

Mangrove endophytic fungi are attracting considerable attention from natural product chemists and biologists alike and a great deal of structurally diverse natural products with unusual biological activity have been discovered recently [1-3]. Fungal genera Alternaria, Aspergillus, Cladosporium, Colletotrichum, Fusarium, Paecilomyces, Penicillium, Pestalotiopsis, Phoma, Phomopsis, Phyllosticta, and Trichoderma are considered as the predominant producer of the most notable bioactive secondary metabolites [4-6]. However, due to the high rediscovery of previously known compounds and scaffolds from these well-investigated organisms, neglected fungal species that are poorly studied might be an alternative source for the discovery of new bioactive compounds.

During our ongoing screening for biologically active secondary metabolites from mangrove endophytic fungi [7-10], we recently investigated Cytospora sp., an endophytic fungus derived from the hypocotyls of Chinese mangrove Ceriops tagal, resulting in the isolation of a new antimicrobial biscyclic sesquiterpene, seiricardine D, together with eight known metabolites [11]. Further study of the aforementioned Cytospora sp. afforded two new polyketides, cytospyrone (1), cytospomarin (2), together with three known metabolites dimethoxyphtalide (3) [12], integracin A (4) [13], and integracin B (5) (Figure 1) [13]. 
<smiles>COc1cc([C@@H](O)[C@@H](C)/C(C)=C/[C@@H](C)/C=C(\C)[C@@H](C)O)oc(=O)c1C</smiles><smiles>COC1=CC2=C(C(=O)OC2C)[C+]1[18O]</smiles>

3<smiles>COc1c(O)cc(O)c2c(=O)oc(C[C@@H](C)O)cc12</smiles>

2

Figure 1. Structures of compounds 1-5.

\section{Results and Discussion}

\subsection{Isolation and Structure Elucidation}

Cytospyrone A (1) was isolated as a white amorphous powder, and has the molecular formula $\mathrm{C}_{20} \mathrm{H}_{30} \mathrm{O}_{5}$ established by high resolution electrospray ionization mass spectroscopy (HR-ESI-MS) at $m / z$ 373.1988 [M + Na] ${ }^{+}$(calculated for 373.1991), indicating six degrees of unsaturation. The ${ }^{1} \mathrm{H}$ and ${ }^{13} \mathrm{C}$ nuclear magnetic resonance (NMR) data of $\mathbf{1}$ in association with distortionless enhancement by polarization transfer (DEPT) and heteronuclear single quantum coherence (HSQC) spectrum suggested the presence of six upfield methyls $\left[\delta_{\mathrm{H}} 1.03,(\mathrm{~d}, J=6.7), \delta_{\mathrm{C}} 21.7, \mathrm{q}, 5^{\prime}-\mathrm{CH}_{3} ; 1.07,(\mathrm{~d}, J=7.0)\right.$, $\delta_{\mathrm{C}} 16.2, \mathrm{q}, 2^{\prime}-\mathrm{CH}_{3} ; 1.18,(\mathrm{~d}, J=6.4), \delta_{\mathrm{C}} 21.9, \mathrm{q}, 9^{\prime}-\mathrm{CH}_{3} ; 1.65, \mathrm{~s}, \delta_{\mathrm{C}} 11.8, \mathrm{q}, 7^{\prime}-\mathrm{CH}_{3} ; 1.67, \mathrm{~s}, \delta_{\mathrm{C}} 10.9$, $\left.\mathrm{q}, 3^{\prime}-\mathrm{CH}_{3} ; 1.85, \mathrm{~s}, \delta_{\mathrm{C}} 8.4, \mathrm{q}, 2-\mathrm{CH}_{3}\right]$, a methoxy group $\left(\delta_{\mathrm{H}} 3.94, \mathrm{~s}, \delta_{\mathrm{C}} 57.3, \mathrm{q}, 4-\mathrm{OCH}_{3}\right)$, three olefinic signals $\left[\delta_{\mathrm{H}} 5.26,(\mathrm{~d}, J=9.0), \delta_{\mathrm{C}} 130.0, \mathrm{~d}, \mathrm{CH}-6^{\prime} ; \delta_{\mathrm{H}} 5.32,(\mathrm{~d}, J=9.1), \delta_{\mathrm{C}} 130.0, \mathrm{~d}, \mathrm{CH}-4^{\prime} ; \delta_{\mathrm{H}} 6.47\right.$, $\left.\mathrm{s}, \delta_{\mathrm{C}} 97.5, \mathrm{~d}, \mathrm{CH}-5\right]$, four methines [2.79, m, $\delta_{\mathrm{C}} 44.1, \mathrm{~d}, 2^{\prime}-\mathrm{CH} ; 3.33-3.42, \mathrm{~m}, \delta_{\mathrm{C}} 32.3, \mathrm{~d}, 5^{\prime}-\mathrm{CH} ; 4.08$, $\left.(\mathrm{d}, J=9.6), \delta_{\mathrm{C}} 80.7, \mathrm{~d}, 1^{\prime}-\mathrm{CH} ; 4.10,(\mathrm{q}, J=6.4), \delta_{\mathrm{C}} 73.9, \mathrm{~d}, 8^{\prime}-\mathrm{CH}\right]$, and six quaternary carbons $\left(\delta_{\mathrm{C}} 101.5\right.$, $134.2,138.1,167.8168 .5,169.1)$. Two substructures, an $\alpha$-pyrone moiety and a linear aliphatic chain, were assigned by analyses of detailed interpretation of ${ }^{1} \mathrm{H}-{ }^{1} \mathrm{H}$ correlation spectroscopy (COSY) and heteronuclear multiple bond correlation (HMBC) correlations (Figure 2). The $\alpha$-pyrone moiety was established by HMBC correlations from $3-\mathrm{CH}_{3}$ to carbons C-2, C-3 and C-4, and from H-5 to C-4 and C-6. Presence of the methoxy group at C-4 was corroborated from the HMBC correlation of its protons $\left(\delta_{\mathrm{H}} 3.94, \mathrm{~s}\right)$ with $\mathrm{C}-4\left(\delta_{\mathrm{C}} 169.1\right)$ and nuclear overhauser effect spectroscopy (NOESY) correlations between $3-\mathrm{CH}_{3} / 4-\mathrm{OCH}_{3}$ and $\mathrm{H}-5 / 4-\mathrm{OCH}_{3}$. A linear aliphatic chain with a thirteen carbon unit was determined by the interpretation of ${ }^{1} \mathrm{H}-{ }^{1} \mathrm{H}$ COSY and HMBC spectroscopic data. The COSY cross-peaks $\left[\mathrm{H}-1^{\prime} / \mathrm{H}-2^{\prime} / 2^{\prime}-\mathrm{CH}_{3}, \mathrm{H}-3^{\prime} / \mathrm{H}-4^{\prime} / 4^{\prime}-\mathrm{CH}_{3} / \mathrm{H}-5^{\prime}, \mathrm{H}-8^{\prime} / \mathrm{H}-9^{\prime}\right]$ and HMBC correlations from $2^{\prime}-\mathrm{CH}_{3}$ to carbons $\mathrm{C}-1^{\prime}, \mathrm{C}-3^{\prime}$, from $3^{\prime}-\mathrm{CH}_{3}$ to carbons $\mathrm{C}-2^{\prime}, \mathrm{C}-3^{\prime}$ and $\mathrm{C}-4^{\prime}$, from $7^{\prime}-\mathrm{CH}_{3}$ to carbons $C-6^{\prime}, C-7^{\prime}$ and $C-8^{\prime}$, and from $\mathrm{H}-9^{\prime}$ to $\mathrm{C}-7^{\prime}$ permitted the construction of a linear aliphatic chain. The above two structural fragments were finally connected to construct the planar structure of compound 1 from the key HMBC correlations from $\mathrm{H}-1^{\prime}$ and $\mathrm{H}-2^{\prime}$ to $\mathrm{C}-6$, from $\mathrm{H}-5$ to $\mathrm{C}-2^{\prime}$. The relative configuration of 1 was deduced by analysis of the NOESY data. The NOESY correlations (Figure 3) between $\mathrm{H}-1^{\prime} / 2^{\prime}-\mathrm{CH}_{3}, 2^{\prime}-\mathrm{CH}_{3} / \mathrm{H}-4^{\prime}, \mathrm{H}-2^{\prime} / 3^{\prime}-\mathrm{CH}_{3}, \mathrm{H}-4^{\prime} / \mathrm{H}-5^{\prime}, \mathrm{H}-5^{\prime} / \mathrm{H}-6^{\prime}, 7^{\prime}-\mathrm{CH}_{3} / \mathrm{H}-8^{\prime}$ and H-6 $/ \mathrm{H}-8^{\prime}$ 
suggested that they were positioned on the same face and both $\Delta^{4^{\prime}}$ and $\Delta^{6^{\prime}}$ double bonds were assigned to the E-configuration. In addition, the correlations of $\mathrm{H}-1^{\prime} / 3^{\prime}-\mathrm{CH}_{3}, 3^{\prime}-\mathrm{CH}_{3} / 5^{\prime}-\mathrm{CH}_{3}$ and $5^{\prime}-\mathrm{CH}_{3} / 7^{\prime}-\mathrm{CH}_{3}$ revealed they were positioned on the other face. To define its absolute configuration, the time dependent density functional theory-(TDDFT)-calculated electronic circular dichroism (ECD) spectrum at the B3LYP/6-31+g $(\mathrm{d}, \mathrm{p})$ level was compared with the experimental circular dichroism (CD) spectrum (Figure 4). Therefore, the structure of 1 was established as 6-((1S, 2S, 3E, 5R, $6 E, 8 R)$-1,8-dihydroxy-2,3,5,7-tetramethylnona-3,6-dienyl)-4-methoxy-3-methyl-2H-pyran-2-one.

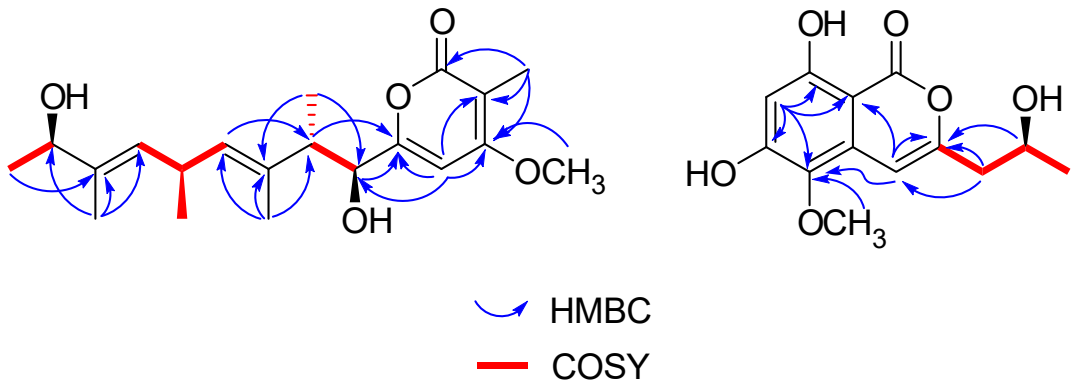

Figure 2. Key $\mathrm{HMBC},{ }^{1} \mathrm{H}-{ }^{1} \mathrm{H}$ COSY correlations of $\mathbf{1}$ and 2.

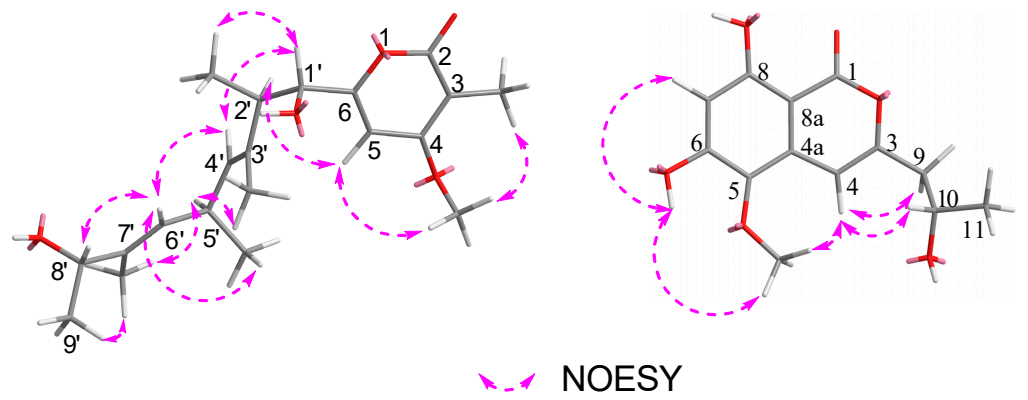

Figure 3. Key NOESY correlations of $\mathbf{1}$ and 2.
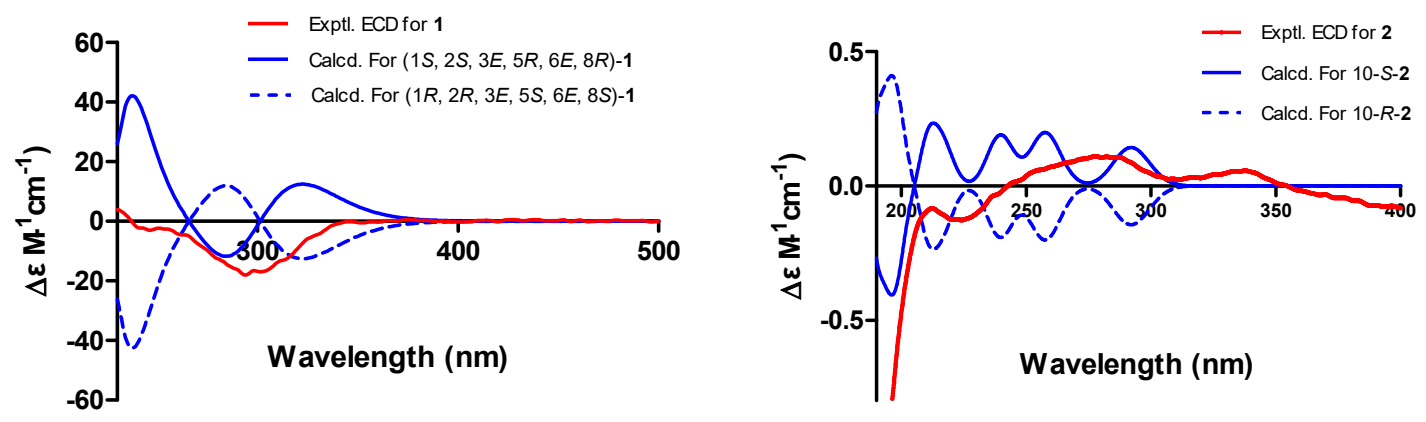

Figure 4. Experimental and calculated electronic circular dichroism (ECD) spectra of $\mathbf{1}$ and $\mathbf{2}$.

Cytospomarin (2), a yellow amorphous solid, was found to have the molecular formula $\mathrm{C}_{13} \mathrm{H}_{14} \mathrm{O}_{6}$, established by HR-ESI-MS $\left(m / z 289.0674\right.$, calculated for $\left.[\mathrm{M}+\mathrm{Na}]^{+} 289.0688\right)$, implying seven degrees of unsaturation. The ${ }^{1} \mathrm{H}$ and ${ }^{13} \mathrm{C}$ NMR data of 2 indicated that six of the seven units of unsaturation could be due to an aromatic ring, a double bond, and one carbonyl group. Thus, the remaining unit of unsaturation was attributed to a ring formation. The ultraviolet (UV) absorption maxima at 247 and $318 \mathrm{~nm}$ suggested 2 is an isocoumarin derivative. The ${ }^{1} \mathrm{H}$ and ${ }^{13} \mathrm{C}$ NMR data of 2 and the DEPT and HSQC spectra revealed the presence of two methyl groups, including a methoxy group $\left(\delta_{\mathrm{H}} 3.68, \mathrm{~s}, \delta_{\mathrm{C}} 60.4, \mathrm{q}, 5-\mathrm{OCH}_{3}\right)$ and a terminal alkyl methyl $\left[\delta_{\mathrm{H}} 1.12,(\mathrm{~d}, J=6.2), \delta_{\mathrm{C}} 23.3\right.$, q, 11- $\left.\mathrm{CH}_{3}\right]$, a methylene $\left(\delta_{\mathrm{H}} 2.50-2.57, \mathrm{~m}, \delta_{\mathrm{C}} 42.8, \mathrm{t}, \mathrm{CH}_{2}-9\right]$, two olefinic methines [ $\delta_{\mathrm{H}} 6.33, \mathrm{~s}, \delta_{\mathrm{C}} 102.6$, $\left.\mathrm{d}, \mathrm{CH}-7 ; \delta_{\mathrm{H}} 6.53, \mathrm{~s}, \delta_{\mathrm{C}} 99.9, \mathrm{~d}, \mathrm{CH}-4\right]$, an oxymethine $\left(\delta_{\mathrm{H}} 3.92-4.00, \mathrm{~m}, \delta_{\mathrm{C}} 64.0, \mathrm{~d}, \mathrm{CH}-10\right)$ and seven quaternary carbons, including a carbonyl $\left(\delta_{C} 165.6, \mathrm{~s}, \mathrm{C}-1\right)$. The ${ }^{1} \mathrm{H}$ and ${ }^{13} \mathrm{C}$ NMR and HSQC 
spectra of compound 2 closely resembled those of an isocoumarin compound peniisocoumarin G [14], recently isolated from another endophytic fungus, Penicillium commune QQF-3, obtained from fresh fruit of the mangrove plant Kandelia candel, indicating that 2 might have the same basic molecular framework as peniisocoumarin G. The only difference between these was replacement of the hydroxyl substituent of peniisocoumarin G by a methoxy group in 2. The HMBC and NOESY correlations (Figures 2 and 3) supported the assignments of the methoxy group $5-\mathrm{OCH}_{3}\left(\delta_{\mathrm{H}} 3.68, \mathrm{~s}\right)$ at $\mathrm{C}-5$ $\left(\delta_{C}\right.$ 134.3). The configuration of $\mathrm{C}-10$ was determined by comparison of optical rotation with the known compounds peniisocoumarin G [14] and botryosphaerin A [15]. The result indicated that compound $\mathbf{2}$ has the same positive value as those two isocoumarins. It was confirmed by comparing experimental and calculating ECD spectra and optical rotation $\left([\alpha]^{20}{ }_{D}=+11.7^{\circ}(\mathrm{c} 0.3, \mathrm{MeOH})\right.$; calculated for $[\alpha]^{20} \mathrm{D}=+89.6^{\circ}(\mathrm{MeOH})$ ) of 2 using TDDFT (Figure 4). Accordingly, compound $\mathbf{2}$ was determined as (S)-6,8-dihydroxy-3-(2-hydroxypropyl)-5-methoxy-1H-isochromen-1-one and named as cytospomarin.

\subsection{Bioactivities}

Compounds 1-5 were testing the in vitro antimicrobial activity against four human pathogens Escherichia coli GIM1.201, methicillin-resistant Staphylococcus aureus GIM1.771 (MRSA), Pseudomonas aeruginosa GIM1.200, Candida albicans GIM2.169, together with three plant pathogens including Bacillus subtilis, Colletotrichum gloeosporioides and Magnaporthe oryze, and the results are summarized in Table 1. Compound 1-5 were also tested in vitro against the human cancer cell line HepG2 with 5-fluorouracil as the positive control. Among them, compound 2 exhibited weak inhibitory activity against $E$. coli GIM1.201 (minimum inhibitory concentration (MIC) $=0.35 \mathrm{mM}$ ) and M. oryzae $(\mathrm{MIC}=1.41 \mathrm{mM})$. Compounds 4 and 5 displayed significant cytotoxicity against human cancer cell line HepG2 with $\mathrm{IC}_{50}$ values of $5.98 \pm 0.12 \mu \mathrm{M}$ and $9.97 \pm 0.06 \mu \mathrm{M}$, respectively, more potent than the positive control 5-fluorouracil $\left(\mathrm{IC}_{50}=43.50 \pm 3.69 \mu \mathrm{M}\right)$ (Table 2).

Table 1. Antimicrobial activities of compounds 1-5.

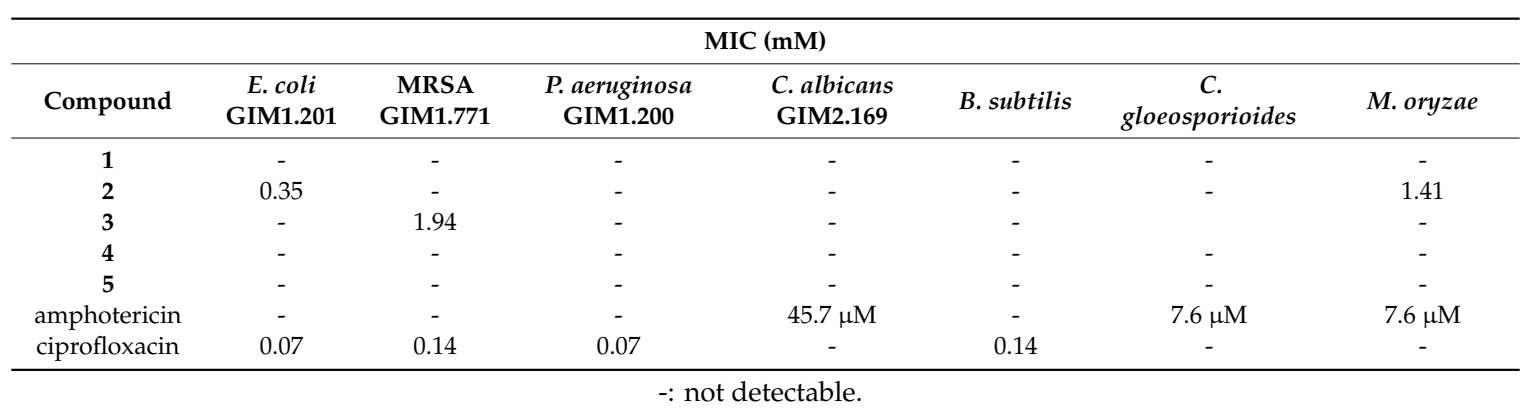

Table 2. Cytotoxicity data of compounds $\mathbf{1}-\mathbf{5} .^{\text {a }}$

\begin{tabular}{cccccc}
\hline Cell Line & \multicolumn{5}{c}{ Compound } \\
\hline & $\mathbf{1 - 2}$ & $\mathbf{3}^{\mathrm{a}}$ & $\mathbf{4}^{\mathrm{a}}$ & $\mathbf{5}^{\mathrm{a}}$ & $\mathbf{5 - F U}^{\mathrm{a}}$ \\
HepG2$\left(\mathrm{IC}_{50}\right)$ & - & $297.97 \pm 17.54$ & $5.98 \pm 0.12$ & $9.97 \pm 0.06$ & $43.50 \pm 3.69$ \\
\hline \multicolumn{5}{c}{ Results are expressed as $\mathrm{IC}_{50}$ values of mean $\pm \mathrm{SD}(n=3)$ in $\mu \mathrm{M}}$.
\end{tabular}

Mangrove endophytic fungi have been proved to be particularly productive with regard to the accumulation of diverse biological natural products and yielded more than 800 natural products [16]. In our previous study, over 120 different compounds, with thirty of them being new polyketides, were discovered from mangrove endophytic fungi, including pyrones [17], isocoumarins [9,17], coumarins [18], cytosporones [18] and chromones [19], etc. In the current study, two new polyketides, cytospyrone (1), cytospomarin (2), together with three known metabolites (3-5) were isolated from the rice culture of Cytospora sp. from Chinese mangrove Ceriops tagal. Structurally, $\mathbf{1}$ and pestalotiopyrones A-H [17] are pyrone derivatives, having both a methoxy group positioned at C-4 and a substituted alkyl side chain at C-6, which all proved to be devoid of significant activity in the antimicrobial and 
cytotoxic bioassays. Many naturally occurring isocoumarin and 3,4-dihydroisocoumarin derivatives are known to have substituents at C-3 and have been shown to possess an impressive array of biological activities [20]. Five dihydroisocoumarins isolated from Cytospora eucalypticola KC1636 [21] exhibited mildly antifungal and antibacterial activities towards Gram positive bacteria, which is similar to our biological finding on $\mathbf{2}$. Compound $\mathbf{3}$ is closely related to 5-dehydroxycytosporone $\mathrm{E}$ previously reported from another Cytospora sp. TT-10, both featuring a phthalan-1-one skeleton [22]. Previously other fungal cytosporone derivatives that bear structural similarities to 3 isolated in our study had been described to be cytotoxic against several cancer cell lines in vitro [23], in contrast to the previous report, 3 showed no activity towards the bioassays we investigated. Integracins A (4) and B (5), exhibited potent (human immunodeficiency virus Type 1) HIV-1 integrase inhibitors, and were dimeric alkyl aromatic compounds isolated for the first time from Cytonaema sp. living in twigs of Quercus ilex [13]. They were later found to be produced by various organisms, such as endophytic fungi Cytospora sp. [22], Chinese mangrove plant Sonneratia hainanensis [24] and Laguncularia racemose [25]. Moreover, compound 4 was also reported to possess potent cytotoxicity against the tumor cell lines HepG2 and NCI-H460 with both $100 \%$ inhibitions at $25 \mathrm{mg} / \mathrm{mL}$ [24]. In this work, we found not only 4 but 5 strongly inhibited the proliferation of human cancer cell line HepG2, which was in accordance with previous reports. This highlighted the high potential of bioprospecting of polyketides from mangrove endophytic fungi Cytospora.

\section{Materials and Methods}

\subsection{General Experimental Procedures}

Optical rotations were measured on a WYA-2S digital Abbe refractometer (Shanghai Physico-optical Instrument Factory, Shanghai, China). UV spectra were obtained on a Shimadzu UV-2401 PC spectrophotometer (Shimadzu Corporation, Tokyo, Japan). Then, 1D and 2D NMR spectra were recorded on Bruker AV 400 NMR apparatus (Bruker Biospin Group, Karlsruhe, Germany) in $\mathrm{CD}_{3} \mathrm{OD}$, DMSO- $d_{6}$ or $\mathrm{CDCl}_{3}$ (Guangzhou Chemical Reagent Factory, Guangzhou, China). Mass spectra were obtained on a LTQ Orbitrap XL instrument (Thermo Fisher Scientific, Bremen, Germany) using peak matching. Column chromatography was performed with silica gel (200-300 mesh, Qingdao Haiyang Chemical Co., Qingdao, China) and Sephadex LH-20 (18-110 $\mu \mathrm{m}$, Merck, Darmstadt, Germany). Semi-preparative HPLC was achieved on an Agilent 1100.

\subsection{Fungal Material and Identification}

Following standard procedures [26], endophytic fungi were isolated from fresh, healthy hypocotyls of Ceriops tagal (Rhizophoraceae), which were collected in October 2015 in Dong Zhai Gang-Mangrove Garden on Hainan Island, China. The fungus (strain no. JGM-9) was identified as Cytospora sp. (GenBank accession no. MG948459) according to DNA amplification and sequencing of the internal transcribed spacer (ITS) region. A voucher strain was deposited at one of the authors' laboratory (J.X.).

\subsection{Fermentation, Extraction and Isolation}

The fungus was grown on solid rice medium at room temperature in $1000 \mathrm{~mL}$-Erlenmeyer flasks containing $100 \mathrm{~g}$ rice (Jiangxi COFCO Rice Industry Co., Nanchang, China) and $100 \mathrm{~mL}$-seawater. After 39 days of cultivation, the mycelia and solid rice medium were extracted with ethyl acetate (EtOAc). The extract was evaporated under reduced pressure to yield $90.0 \mathrm{~g}$ residue. This residue was subjected to silica gel column chromatography (CC) employing a step gradient of petroleum ether/ethyl-acetate gradient (100:0 to 0:100) to obtain four fractions (Fr. 1-Fr. 7). Promising Fr. 5 was separated by CC (silica gel; petroleum ether/ethyl acetate/acetone 5:2:2) to give four sub-fractions. Sub-Fr. 5-2 was purified with silica gel by $\mathrm{CHCl}_{3} / \mathrm{MeOH}$ 20:1 and reversed-phase-HPLC $\left(\mathrm{MeOH} / \mathrm{H}_{2} \mathrm{O}\right.$ 80:20) to yield compound 2 (15.0 mg). Sub-Fr. 5-3 was subjected to CC (Sephadex LH-20, $\mathrm{MeOH} / \mathrm{CHCl}_{3}$ 1:1) and reversed-phase-HPLC ( $\mathrm{MeOH} / \mathrm{H}_{2} \mathrm{O} 70: 30$ to 100:0) to furnish $\mathbf{1}(8.1 \mathrm{mg}), \mathbf{4}(5.7 \mathrm{mg})$ and 5 (3.8 mg). 
Fr. 7 was subjected to CC (silica gel; gradient petroleum ether / acetone) and reversed-phase-HPLC $\left(\mathrm{MeOH} / \mathrm{H}_{2} \mathrm{O}\right.$ 80:20 to 100:0) to afford 3 (3.8 mg).

Cytospyrone (1): white amorphous powder $(\mathrm{MeOH}) ;[\alpha]^{20} \mathrm{D}=-23.1^{\circ}(\mathrm{c} 0.05, \mathrm{MeOH}) ; \mathrm{UV}(\mathrm{MeOH})$ $\lambda_{\max } 212 \mathrm{~nm} ;{ }^{1} \mathrm{H}$ NMR(400 MHz, CD $\left.{ }_{3} \mathrm{OD}\right) \delta_{\mathrm{H}} 6.47(1 \mathrm{H}, \mathrm{s}, \mathrm{H}-5), 5.32\left(1 \mathrm{H}, \mathrm{d}, J=9.1 \mathrm{~Hz}, \mathrm{H}-4^{\prime}\right)$, $5.29\left(1 \mathrm{H}, \mathrm{d}, J=9.0,6^{\prime}\right), 4.10\left(1 \mathrm{H}, \mathrm{q}, J=6.4, \mathrm{H}-8^{\prime}\right), 4.08\left(1 \mathrm{H}, \mathrm{d}, J=9.6, \mathrm{H}-1^{\prime}\right), 3.94\left(3 \mathrm{H}, \mathrm{s}, 4-\mathrm{OCH}_{3}\right)$, 3.33-3.42 $\left(1 \mathrm{H}, \mathrm{m}, \mathrm{H}-5^{\prime}\right), 2.79\left(1 \mathrm{H}, \mathrm{m}, \mathrm{H}-2^{\prime}\right), 1.85\left(3 \mathrm{H}, \mathrm{s}, 3-\mathrm{CH}_{3}\right), 1.67\left(3 \mathrm{H}, \mathrm{s}, 3^{\prime}-\mathrm{CH}_{3}\right), 1.65(3 \mathrm{H}$, $\left.\mathrm{s}, 7^{\prime}-\mathrm{CH}_{3}\right), 1.18\left(3 \mathrm{H}, \mathrm{d}, J=6.4, \mathrm{H}-9^{\prime}\right), 1.07\left(3 \mathrm{H}, \mathrm{d}, J=7.0,2^{\prime}-\mathrm{CH}_{3}\right), 1.03\left(3 \mathrm{H}, \mathrm{d}, J=6.7,5^{\prime}-\mathrm{CH}_{3}\right)$; ${ }^{13} \mathrm{C}-\mathrm{NMR}\left(100 \mathrm{MHz}, \mathrm{CD}_{3} \mathrm{OD}\right) \delta_{\mathrm{C}} 169.1$ (C-4), 168.5 (C-2), 167.8 (C-6), 138.1 (C-7'), $135.3\left(\mathrm{C}-4^{\prime}\right), 134.2$ (C-3'), $130.1\left(\mathrm{C}-6^{\prime}\right), 101.5(\mathrm{C}-3), 97.5(\mathrm{C}-5), 80.7\left(\mathrm{C}-1^{\prime}\right), 73.9\left(\mathrm{C}-8^{\prime}\right), 57.3\left(4-\mathrm{OCH}_{3}\right), 44.1\left(\mathrm{C}-2^{\prime}\right), 32.3\left(\mathrm{C}-5^{\prime}\right)$, $21.9\left(\mathrm{C}-9^{\prime}\right), 21.7\left(5^{\prime}-\mathrm{CH}_{3}\right), 16.2\left(3^{\prime}-\mathrm{CH}_{3}\right), 11.8\left(7^{\prime}-\mathrm{CH}_{3}\right), 10.8\left(3^{\prime}-\mathrm{CH}_{3}\right), 8.4\left(3-\mathrm{CH}_{3}\right) ; \mathrm{HR}-\mathrm{ESI}-\mathrm{MS} \mathrm{m} / \mathrm{z}$ $373.1991[\mathrm{M}+\mathrm{Na}]^{+}$(calculated for $\mathrm{C}_{20} \mathrm{H}_{30} \mathrm{O}_{5} \mathrm{Na}$, 373.1991) (Supplementary Materials).

Cytospomarin (2): a yellow amorphous solid $(\mathrm{MeOH}) ;[\alpha]^{20}{ }_{\mathrm{D}}=+11.7^{\circ}$ (c 0.3, MeOH); $\mathrm{UV}(\mathrm{MeOH}) \lambda_{\max } 318 \mathrm{~nm}, 247 \mathrm{~nm} ;{ }^{1} \mathrm{H}$ NMR $\left(400 \mathrm{MHz}\right.$, DMSO-d $\left.{ }_{6}\right) \delta_{\mathrm{H}} 10.8(1 \mathrm{H}$, br s, 6-OH), $6.53(1 \mathrm{H}, \mathrm{s}, \mathrm{H}-4), 6.33(1 \mathrm{H}, \mathrm{s}, \mathrm{H}-7), 3.92-4.00(1 \mathrm{H}, \mathrm{m}, \mathrm{H}-10) 3.68\left(3 \mathrm{H}, \mathrm{s}, 5-\mathrm{OCH}_{3}\right), 2.50-2.57(2 \mathrm{H}, \mathrm{m}, \mathrm{H}-9)$, $1.12(3 \mathrm{H}, \mathrm{d}, J=6.2, \mathrm{H}-11) ;{ }^{13} \mathrm{C}-\mathrm{NMR}\left(100 \mathrm{MHz}, \mathrm{DMSO}-d_{6}\right) \delta_{\mathrm{C}} 165.6(\mathrm{C}-1), 161.1$ (C-8), 158.6 (C-6), 154.7 (C-3), 134.3 (C-5), 130.3 (C-5a), 102.6 (C-7), 99.9 (C-4), 95.5 (C-8a), 64.0 (C-10), $60.4\left(5-\mathrm{OCH}_{3}\right)$, 42.8 (C-9), 23.3 (C-11); HRESIMS $\mathrm{m} / 2289.0674$ [M + Na] $]^{+}$(calculated for $\mathrm{C}_{13} \mathrm{H}_{14} \mathrm{O}_{6} \mathrm{Na}, 289.0610$ ).

Dimethoxyphtalide (3): white amorphous powder $(\mathrm{MeOH}) ;[\alpha]^{20} \mathrm{D}=+123^{\circ}(\mathrm{c} 0.1, \mathrm{MeOH})$, $\mathrm{UV}(\mathrm{MeOH}) \lambda_{\max } 216 \mathrm{~nm},{ }^{1} \mathrm{H}$ NMR $\left(400 \mathrm{MHz}, \mathrm{CDCl}_{3}\right) \delta_{\mathrm{H}} 6.55(1 \mathrm{H}, \mathrm{s}, \mathrm{H}-4), 5.40(1 \mathrm{H}, \mathrm{d}, J=6.6, \mathrm{H}-3)$, $4.05\left(3 \mathrm{H}, \mathrm{s}, 7-\mathrm{OCH}_{3}\right), 3.92\left(3 \mathrm{H}, \mathrm{s}, 5-\mathrm{OCH}_{3}\right), 2.14\left(3 \mathrm{H}, \mathrm{s}, 6-\mathrm{CH}_{3}\right), 1.59\left(3 \mathrm{H}, \mathrm{d}, J=6.6,3-\mathrm{CH}_{3}\right) ;{ }^{13} \mathrm{C}-\mathrm{NMR}$ $\left(100 \mathrm{MHz}, \mathrm{CDCl}_{3}\right) \delta_{\mathrm{C}} 168.3$ (C-1), 164.2 (C-5), 157.6 (C-7), 152.9 (C-3a), 120.5 (C-6), 109.7 (C-7a), 97.9 (C-4), $76.4(\mathrm{C}-3), 62.1\left(7-\mathrm{OCH}_{3}\right), 56.1\left(5-\mathrm{OCH}_{3}\right), 29.7$ (C-9), 20.7 (C-8); HRESIMS m/z 233.0969 $[\mathrm{M}+\mathrm{H}]^{+}$(calculated for $\mathrm{C}_{12} \mathrm{H}_{15} \mathrm{O}_{4}, 233.0969$ ). The data of ${ }^{1} \mathrm{H}$ NMR and ${ }^{13} \mathrm{C}$ NMR are basically consistent with those reported in literature [12].

Integracin A (4): yellow oil $(\mathrm{MeOH}) ;[\alpha]^{20}{ }_{\mathrm{D}}=+97.0^{\circ}$ (c $\left.0.1, \mathrm{MeOH}\right) ; \mathrm{UV}(\mathrm{MeOH}) \max$ 280,259,218 nm; ${ }^{1} \mathrm{H}$ NMR(500 MHz, CD3OD) $6.23\left(\mathrm{H}, \mathrm{d}, J=1.8, \mathrm{H}-6^{\prime}\right), 6.19\left(\mathrm{H}, \mathrm{d}, J=1.8, \mathrm{H}-4^{\prime}\right)$, $6.14(2 \mathrm{H}, \mathrm{d}, J=1.4, \mathrm{H}-1, \mathrm{H}-5), 6.10(\mathrm{H}, \mathrm{br} \mathrm{s}, \mathrm{H}-3), 5.28(\mathrm{H}$, pent, $J=5.6 \mathrm{~Hz}, \mathrm{H}-14), 4.82(\mathrm{H}$, overlapped, $\left.\mathrm{H}-15^{\prime}\right), 2.81-2.85\left(2 \mathrm{H}, \mathrm{m}, \mathrm{H}-8^{\prime}\right), 2.45(\mathrm{H}, \mathrm{t}, J=8.0 \mathrm{~Hz}, \mathrm{H}-7), 2.03\left(3 \mathrm{H}, \mathrm{s}, \mathrm{Me}-2^{\prime \prime}\right), 1.66-1.76(4 \mathrm{H}, \mathrm{m}$, H-13, H-15), 1.54-1.57 (4H, m, H-8, H-9'), 1.40-1.42 (4H, m, H-14' $\left.{ }^{\prime}, \mathrm{H}-16^{\prime}\right), 1.31-1.35$ (20H, m, 9-H to $12-\mathrm{H}, \mathrm{H}-16, \mathrm{H}-10^{\prime}$ to $\left.\mathrm{H}-13^{\prime}, \mathrm{H}-17^{\prime}\right), 0.99\left(3 \mathrm{H}, \mathrm{t}, J=7.3 \mathrm{~Hz}, \mathrm{Me}-18^{\prime}\right), 0.94(3 \mathrm{H}, \mathrm{t}, J=7.3, \mathrm{Me}-17)$;

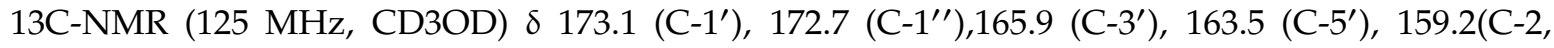
C-4), 149.0 (C-7'), 146.3 (C-6), 111.9 (CH-6'), 107.9 (CH-1, CH-5), 105.8 (C-2'), 101.9 (CH-4'), 101.0 (CH-3), 76.7 (CH-14), 75.5 (CH-15'), 37.7 a (CH2-8'), 37.4 a (CH2-15), 37.4 a (CH2-16'), 36.9 (CH2-7), 35.2 (CH2-13), 35.2 (CH2-14'), 33.6 (CH2-9'), $32.3(\mathrm{CH} 2-8), 31.0$ a (CH2-10'), 30.7 a $\left(\mathrm{CH} 2-11^{\prime}\right), 30.5$ a (CH2-12'), 30.5 a (CH2-11), 30.4 a (CH2-10), 30.2 (CH2-9), 26.6 (CH2-13'), 26.4 (CH2-12), 21.2 (CH3-2' '), 19.9 (CH2-16), 19.6 (CH2-17'), 14.3 (CH3-18'), 14.2 (CH3-17), chemical shifts could be interchanged; ESI-MS $[\mathrm{M}+\mathrm{H}]+m / z 629.4$ (C37H57O8). The data of $1 \mathrm{H}$ NMR and 13C NMR are basically consistent with those reported in literature [13].

Integracin B (5): yellow oil $(\mathrm{MeOH}) ;[\alpha] 20 \mathrm{D}=+37.8^{\circ}$ (c 0.30, MeOH); UV (MeOH) $\lambda_{\max } 300$, 264, $230 \mathrm{~nm}$; 1H NMR(500 MHz, CD3OD) $\delta 11.98\left(\mathrm{H}, \mathrm{s}, 3^{\prime}-\mathrm{OH}\right), 7.61(\mathrm{H}, \mathrm{br} \mathrm{s}, 3 \times-\mathrm{OH}), 6.21(\mathrm{H}, \mathrm{d}$, $\left.J=2.4, \mathrm{H}-6^{\prime}\right), 6.19\left(\mathrm{H}, \mathrm{d}, J=2.4 \mathrm{~Hz}, \mathrm{H}-4^{\prime}\right), 6.14(2 \mathrm{H}, \mathrm{d}, J=2.1, \mathrm{H}-1, \mathrm{H}-5), 6.10(\mathrm{H}, \mathrm{t}, J=2.1, \mathrm{H}-3)$, $5.20(\mathrm{H}$, pent, $J=5.7, \mathrm{H}-14), 3.48-3.52\left(\mathrm{H}, \mathrm{m}, \mathrm{H}-15^{\prime}\right), 2.80\left(2 \mathrm{H}, \mathrm{td}, J=7.0,2.5, \mathrm{H}-8^{\prime}\right), 2.40(\mathrm{H}, \mathrm{t}, J=7.5$, H-7), 1.63-1.67 (2H, m, H-13), 1.59-1.63 (2H, m, H-15), 1.48-1.55 (4H, m, H-8, H-9'), 1.36-1.39 (4H, m, H-14', H-16' $), 1.34-1.36$ (2H, m, H-17'), 1.28-1.30 (2H, m, H-16), 1.23-1.28 (20H, m, 9-H to 12-H, H-16, $\mathrm{H}-10^{\prime}$ to $\left.\mathrm{H}-13^{\prime}, \mathrm{H}-17^{\prime}\right), 0.91$ ( $3 \mathrm{H}, \mathrm{t}, J=7.3$, Me-18'), 0.88 (3H, t, $\left.J=6.8, \mathrm{Me}-17\right) ; 13 \mathrm{C}-\mathrm{NMR}(125 \mathrm{MHz}$, CD3OD) $\delta 172.3\left(\mathrm{C}-1^{\prime}\right), 165.5$ (C-3'), 162.8 (C-5'), 158.4(C-2, C-4), 149.1 (C-7'), 146.1 (C-6), 111.8 (CH-6'), 107.8 (CH-1, CH-5), 105.1(C-2'), 101.6 (CH-4' ), 100.6 (CH-3), 76.3 (CH-14), 71.9 (CH-15'), $40.0\left(\mathrm{CH} 2-16^{\prime}\right)$, 37.9 (CH2-8'), 37.6 (CH2-15), 37.2 (CH2-7), 36.6 (CH2-13), 35.0 (CH2-14'), 33.1 (CH2-9'), 31.9 (CH2-8), 30.7 a (CH2-10'), 30.5 a (CH2-11'), 30.4 a (CH2-12'), 30.1 a (CH2-11), 30.0 a (CH2-10), 29.9 (CH2-9), 26.4 (CH2-13'), 26.2 (CH2-12), 19.5 (CH2-16), 19.4 (CH2-17'), 14.4 (CH3-18'), 14.3 (CH3-17), a chemical 
shifts could be interchanged; ESIMS [M + H]+ m/z 587.4 (C35H55O7). The data of 1H NMR and 13C NMR are basically consistent with those reported in literature [13].

\subsection{ECD Calculations}

Monte Carlo conformational searches were carried out by means of Spartan 14 software using Merck molecular force field (MMFF). The conformers with Boltzmann population of over $1 \%$ were chosen for ECD calculations, and then the conformers were initially optimized at B3LYP/6-31g level in gas. The theoretical calculation of ECD was conducted in $\mathrm{MeOH}$ using time dependent density functional theory (TDDFT) at the B3LYP/6-31+g $(\mathrm{d}, \mathrm{p})$ level for all conformers of compounds 1 and 2. ECD spectra were generated using the program SpecDis 1.6 (University of Würzburg, Würzburg, Germany) and GraphPad Prism 5 (University of California, San Diego, CA, USA) from dipole-length rotational strengths by applying Gaussian band shapes with sigma $=0.3 \mathrm{eV}$.

\subsection{Optical Rotation (OR) Calculations}

Monte Carlo conformational searches were carried out by means of Spartan 10 software using Merck molecular force field (MMFF). The conformers with Boltzmann population of over $1 \%$ were chosen for OR calculations, and then the conformers were initially optimized at B3LYP/6-31+g $(d, p)$ level in $\mathrm{MeOH}$ using the conductor-like polarizable continuum model (CPCM) polarizable conductor calculation model. The theoretical calculation of OR was conducted in $\mathrm{MeOH}$ using time dependent density functional theory (TDDFT) at the B3LYP/6-31+g (d, p) level for all conformers of compound 2 .

\subsection{Antimicrobial Activity}

The antimicrobial activity was determined against human pathogens Escherichia coli GIM1.201, methicillin-resistant Staphylococcus aureus GIM1.771 (MRSA), Pseudomonas aeruginosa GIM1.200, Candida albicans GIM2.169 and plant pathogenic fungi Bacillus subtilis, Colletotrichum gloeosporioides and Magnaporthe oryze (Guangdong Microbial Culture Collection Center, China, CDMCC). The bacteria was cultured in Luria-Bertani (LB) medium (10 $\mathrm{g}$ of peptone, $15 \mathrm{~g}$ of sodium chloride, $3 \mathrm{~g}$ of yeast extract, and $1000 \mathrm{~mL}$ of distilled $\left.\mathrm{H}_{2} \mathrm{O}\right)$ at $37^{\circ} \mathrm{C}(160 \mathrm{rpm})$ for $24 \mathrm{~h}$, and the fungi was cultured in potato dextrose broth (PDB) medium (200 g of potatoes, $20 \mathrm{~g}$ of dextrose, and $1000 \mathrm{~mL}$ of distilled $\left.\mathrm{H}_{2} \mathrm{O}\right)$ at $28^{\circ} \mathrm{C}(160 \mathrm{rpm})$ for $48 \mathrm{~h}$. All isolated compounds were subjected to antimicrobial assays using a serial dilution technique, as previously described [26]. The minimum inhibitory concentration (MIC) was assigned to the lowest concentration that completely inhibited the growth of the indicator microorganisms. Ciprofloxacin (Guangzhou Lianghua Chemical Co., Ltd., 99.9\%, Guangzhou, China) and amphotericin (American AMRESCO, 99.9\%, Branded Products Group, Cochran, USA) were used as a positive control.

\subsection{Cytotoxicity Assay}

The human hepatoma cell (HepG2) cells (Cell Resource Center, Shanghai Institutes for Biological Sciences, Chinese Academy of Sciences) were grown in RPMI-1640 culture medium. Cytotoxicity against HepG2 cells was evaluated using the 3-(4,5-dimethylthiazol-2-yl)-2,5-diphenyltetrazolium bromide (MTT) (Sigma-Aldrich, Missouri, St. Louis, MO, USA) method, as described previously [8] and 5-Fluorouracil (5-FU) (Beijing Solarbio Science \& Technology Co., Ltd., 99.8\%) (Beijing, China) was used as the positive control.

\section{Conclusions}

In the present work, two new polyketides cytospyrone (1), cytospomarin (2) and three known metabolites (3-5) were obtained from the ethyl acetate extract of the fermented cultures of the fungus Cytospora sp., which was isolated from the Chinese mangrove Ceriops tagal. Compound $\mathbf{2}$ exhibited 
weak inhibitory activity against Escherichiacoli GIM1.201 and compounds 4 and 5 displayed potent cytotoxicity against human cancer cell line HepG2.

Supplementary Materials: The following are available online, Figure S1. ${ }^{1} \mathrm{H}-\mathrm{NMR}$ of cytospyrone (1); Figure S2. ${ }^{13} \mathrm{C}$-NMR of cytospyrone (1); Figure S3. DEPT of cytospyrone (1); Figure $\mathrm{S} 4 .{ }^{1} \mathrm{H}^{-1} \mathrm{H}$ COSY of cytospyrone (1); Figure S5. HMQC of cytospyrone (1); Figure S6. HMBC of cytospyrone (1); Figure S7. ROESY of cytospyrone (1); Figure S8. HR-ESI-MS of cytospyrone (1); Figure S9 CD spectrum of cytospyrone (1); Figure S10. ${ }^{1} \mathrm{H}-\mathrm{NMR}$ of cytospomarin (2); Figure S11. ${ }^{13} \mathrm{C}-\mathrm{NMR}$ of cytospomarin (2); Figure S12. DEPT of cytospomarin (2); Figure S13. ${ }^{1} \mathrm{H}-{ }^{1} \mathrm{H}$ COSY of cytospomarin (2); Figure S14. HMQC of cytospomarin (2); Figure S15. HMBC of cytospomarin (2); Figure S16. ROESY of cytospomarin (2); Figure S17. HR-ESI-MS of cytospomarin (2); Figure S18. CD spectrum of cytospomarin (2). Table S1. Gibbs free energies ${ }^{a}$ and equilibrium populations $s^{b}$ of low-energy conformers of cytospyrone; Table S2. Cartesian coordinates for the low-energy reoptimized MMFF conformers of cytospyrone (1) at B3LYP/6-31G(d,p) level of theory in gas; Table S3. Gibbs free energies ${ }^{a}$ and equilibrium populations ${ }^{b}$ of low-energy conformers of cytospomarin (2); Table S4. Cartesian coordinates for the low-energy reoptimized MMFF conformers of cytospomarin (2) at B3LYP/6-31G $(\mathrm{d}, \mathrm{p})$ level of theory in gas; Table S5. Gibbs free energiesa and equilibrium populationsb of low-energy conformers of cytospomarin (2); Table S6. Cartesian coordinates for the low-energy reoptimized MMFF conformers of cytospomarin (2) at B3LYP/6-31+G(d,p) level of theory in $\mathrm{CH}_{3} \mathrm{OH}$.

Author Contributions: C.W. and Q.D. isolated the compounds. Q.D. carried out the large-scale fermentation and extraction. M.S. performed antimicrobial and cytotoxic activity evaluation. J.X. designed and supervised this research, structure elucidation and manuscript preparation. J.X. designed and supervised this research. The final revision of the manuscript was revised by all the authors. All authors have read and agreed to the published version of the manuscript.

Funding: This work was financially supported by the High-level Talents Programs of Hainan Province (2019RC006), National Natural Science Foundation of China (no. 81973229/81660584), Key Research and Development Program of Hainan Province (ZDYF2017099). Key Project of Education Department of Hainan Province (Hnky2019ZD-6) and the Key Lab of Tropical Pharmaceutical Herb Chemistry of Hainan Province Open Fund in Hainan Normal University (RDZH2019004) are gratefully acknowledged.

Conflicts of Interest: The authors declare no conflict of interest.

\section{References}

1. Ancheeva, E.; Daletos, G.; Proksch, P. Lead compounds from mangrove-associated microorganisms. Mar. Drugs 2018, 16, 319. [CrossRef] [PubMed]

2. $\mathrm{Xu}, \mathrm{J}$. Bioactive natural products derived from mangrove-associated microbes. RSC Adv. 2015, 5, 841-892. [CrossRef]

3. $\mathrm{Xu}, \mathrm{J}$. Biomolecules produced by mangrove-associated microbes. Curr. Med. Chem. 2011, 18, 5224-5266. [CrossRef] [PubMed]

4. Zhou, J.; Xu, J. Chemistry and biodiversity of Rhizophora-derived endophytic fungi. In Mangrove Ecosystem Ecology and Function; IntechOpen: London, UK, 2018; Volume 8, pp. 165-186.

5. Debbab, A.; Aly, A.H.; Proksch, P. Mangrove derived fungal endophytes-a chemical and biological perception. Fungal Divers. 2013, 61, 1-27. [CrossRef]

6. Deng, Q.; Yang, X.B.; Lv, X.B.; Xu, J. Biomolecules produced by mangrove Ceriops tagal endophytic fungi. Chin. J. Antibiot. 2018, 43, 635-643.

7. $\mathrm{Xu}, \mathrm{Z} . \mathrm{Y}$; Xiong, B.X.; $\mathrm{Xu}, \mathrm{J}$. Chemical investigation of secondary metabolites produced by mangrove endophytic fungus Phyllosticta capitalensis. Nat. Prod. Res. 2019, 1-5. [CrossRef]

8. Zhou, J.; Li, G.; Deng, Q.; Zheng, D.Y.; Yang, X.B.; Xu, J. Cytotoxic constituents from the mangrove endophytic Pestalotiopsis sp. induce $\mathrm{G}(0) / \mathrm{G}(1)$ cell cycle arrest and apoptosis in human cancer cells. Nat. Prod. Res. 2018, 32, 2968-2972. [CrossRef]

9. Xu, Z.Y.; Wu, X.; Li, G.; Feng, Z.; Xu, J. Pestalotiopisorin B, a new isocoumarin derivative from the mangrove endophytic fungus Pestalotiopsis sp. HHL101. Nat. Prod. Res. 2019, 1-6. [CrossRef]

10. Hemberger, Y.; Xu, J.; Wray, V.; Proksch, P.; Wu, J.; Bringmann, G. Pestalotiopens A and B: Stereochemically challenging flexible sesquiterpene-cyclopaldic acid Hybrids from Pestalotiopsis sp. Chem. Eur. J. 2013, 19, 15556-15564. [CrossRef] 
11. Deng, Q.; Li, G.; Sun, M.Y.; Yang, X.B.; Xu, J. A new antimicrobial sesquiterpene isolated from endophytic fungus Cytospora sp. from the Chinese mangrove plant Ceriops tagal. Nat. Prod. Res. 2018, 34, 1404-1408. [CrossRef]

12. Bradamante, S.; Barenghi, L.; Beretta, G.; Bonfa, M.; Rollini, M.; Manzoni, M. Production of lovastatin examined by an integrated approach based on chemometrics and DOSY-NMR. Biotechnol. Bioeng. 2002, 80, 589-593. [CrossRef] [PubMed]

13. Singh, S.B.; Zink, D.L.; Bills, G.F.; Pelaez, F.; Teran, A.; Collado, J.; Silverman, K.C.; Lingham, R.B.; Felock, P.; Hazuda, D.J. Discovery, structure and HIV-1 integrase inhibitory activities of integracins, novel dimeric alkyl aromatics from Cytonaema sp. Tetrahedron Lett. 2002, 43, 1617-1620. [CrossRef]

14. Cai, R.L.; Wu, Y.N.; Chen, S.H.; Cui, H.; Liu, Z.M.; Li, C.Y.; She, Z.G. Peniisocoumarins A-J: Isocoumarins from Penicillium commune QQF-3, an endophytic fungus of the mangrove plant Kandelia candel. J. Nat. Prod. 2018, 81, 1376-1383. [CrossRef] [PubMed]

15. Savi, D.C.; Shaaban, K.A.; Mitra, P.; Ponomareva, L.V.; Thorson, J.S.; Glienke, C.; Rohr, J. Secondary metabolites produced by the citrus phytopathogen Phyllosticta citricarpa. J. Antibiot. 2019, 72, 306-310. [CrossRef]

16. Xu, J. Natural Products of Mangrove-Derived Microbes; Science Press: Beijing, China, 2014; ISBN 97-7-03-041971-2.

17. Xu, J.; Aly, A.H.; Wray, V.; Proksch, P. Polyketide Derivatives of endophytic fungus Pestalotiopsis sp. isolated from the Chinese mangrove plant Rhizophora mucronata. Tetra Lett. 2011, 52, 21-25. [CrossRef]

18. Xu, J.; Kjer, J.; Sendker, J.; Wray, V.; Proksch, P. Cytosporones, coumarins, and an alkaloid from the endophytic fungus Pestalotiopsis sp. isolated from the Chinese mangrove plant Rhizophora mucronata. Bioorg. Med. Chem. 2009, 17, 7362-7367. [CrossRef]

19. Xu, J.; Kjer, J.; Sendker, J.; Wray, V.; Proksch, P. Chromones from the endophytic fungus Pestalotiopsis sp. isolated from the Chinese mangrove plant Rhizophora mucronata. J. Nat. Prod. 2009, 72, 662-665. [CrossRef]

20. Thongbai, B.; Surup, F.; Mohr, K.; Kuhnert, E.; Hyde, K.D.; Stadler, M. Gymnopalynes A and B, chloropropynylisocoumarin antibiotics from cultures of the basidiomycete Gymnopus sp. J. Nat. Prod. 2013, 76, 2141-2144. [CrossRef]

21. Tetsuo, K.; Nigel, C.V.; Paul, D.B.; Monique, S.J.S. Dihydroisocoumarins and a tetralone from Cytospora eucalypticola. Phytochemistry 2003, 62, 779-782.

22. Takano, T.; Koseki, T.; Koyama, H.; Shiono, Y. A new cytosporone derivative from the endophytic fungus Cytospora sp. Nat. Prod. Commun. 2014, 9, 973-975. [CrossRef]

23. Brady, S.F.; Wagenaar, M.M.; Singh, M.P.; Janso, J.E.; Clardy, J. The cytosporones, new octaketide antibiotics isolated from an endophytic fungus. Org. Lett. 2000, 23, 4043-4046. [CrossRef] [PubMed]

24. Liu, H.L.; Huang, X.Y.; Li, J.; Xin, G.R.; Guo, Y.W. Absolute configurations of integracins A, B, and 15'-dehydroxy-integracin B. Chirality 2012, 24, 459-462. [CrossRef] [PubMed]

25. Shi, C.; Xu, M.J.; Bayer, M.; Deng, Z.W.; Kubbutat, M.H.G.; Waejen, W.; Proksch, P.; Lin, W.H. Phenolic compounds and their anti-oxidative properties and protein kinase inhibition from Chinese mangrove plant Laguncularia racemose. Phytochemistry 2010, 71, 435-442. [CrossRef] [PubMed]

26. Zhou, J.; Diao, X.P.; Wang, T.; Chen, G.Y.; Lin, Q.; Yang, X.B.; Xu, J. Phylogenetic diversity and antioxidant activities of culturable fungal endophytes associated with the mangrove species Rhizophora stylosa and R. mucronata in the South China Sea. PLoS ONE 2018, 13, e0197359. [CrossRef]

Sample Availability: Samples of the compounds are not available from the authors. 